\title{
Nível de atividade física em mulheres idosas e fatores associados
}

\section{Level of physical activity in elderly women and associated factors}

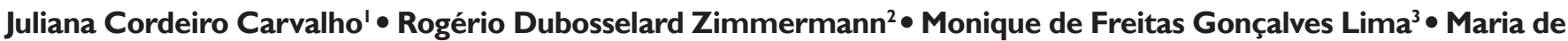 \\ Fatima de Oliveira Falcão ${ }^{4}$ Anna Carolina de Sena e Vasconcelos ${ }^{5}$ Suelane Renata de Andrade Silva ${ }^{6}$
}

\begin{abstract}
RESUMO
O objetivo da pesquisa foi avaliar o nível de atividade física em mulheres idosas e seus fatores associados. Trata-se de um estudo observacional-descritivo, quantitativo de corte transversal. Foram avaliadas 110 idosas cadastradas nos cursos de línguas pela Universidade Aberta da Terceira Idade (UNATI) inserida na Universidade Federal de Pernambuco (UFPE), no município do Recife-PE. Foram analisado o nível de atividade física, a função e satisfação sexual, a imagem corporal, condições sociodemográficas e clínicas.Verificou-se que $82,7 \%$ das idosas foram consideradas ativas, a maioria em atividades domésticas, enquanto as atividades físicas no trabalho foram a com pior nível ( $90 \%$ das idosas eram inativas). A maioria das mulheres idosas que tinham um bom nível de atividade física (AF), não ingeriam bebidas alcoólicas e não fumavam, ocorrendo relação entre as variáveis AF, uso de álcool e do cigarro. Concluiu-se que a ausência do uso de substâncias psicoativas no estilo de vida dessas idosas acarretou em um bom nível de atividade física.
\end{abstract}

Palavras Chaves: Idosa,Atividade Física. Socioeconômicos. Fatores Epidemiológicos. Sexualidade. Imagem Corporal.

\begin{abstract}
The objective of the research was to evaluate the level of physical activity in elderly women and their associated factors. This is an observational-descriptive, quantitative cross-sectional study. I 10 elderly women enrolled in language courses by the Open University of the Third Age (UNATI) at the Federal University of Pernambuco (UFPE), in the city of Recife-PE, were evaluated. The level of physical activity, sexual function and satisfaction, body image, sociodemographic and clinical conditions were analyzed. It was found that $82.7 \%$ of the elderly women were considered active, the majority in domestic activities, while physical activities at work were the worst ( $90 \%$ of the elderly women were inactive). Most elderly women who had a good level of physical activity (PA), did not drink alcohol and did not smoke, with a relationship between the variables PA, alcohol and cigarette use. It was concluded that the absence of the use of psychoactive substances in the lifestyle of these elderly women resulted in a good level of physical activity.

Keywords: Elderly, Physical Activity. Socioeconomic Factors. Epidemiological Factors. Sexuality. Body Image.
\end{abstract}




\section{INTRODUÇÃO}

O envelhecimento é definido como um processo gradual, universal e irreversível, provocando uma perda funcional progressiva no organismo. Esse processo é caracterizado por diversas alterações orgânicas, como a redução do equilíbrio e da mobilidade, das capacidades fisiológicas e modificações psicológicas( ${ }^{(1)}$.

A promoção da saúde e a prevenção de doenças nos idosos tornam-se cada vez mais importantes uma vez que, paralelamente às alterações demográficas, ocorre o aumento da expectativa de vida e, consequentemente, também o aumento do risco e da incidência de doenças crônicas degenerativas ${ }^{(2)}$.

$\mathrm{Na}$ população idosa, a prática de AF gera inúmeros benefícios, e tem por objetivo evitar, minimizar, e/ou reverter muitos dos declínios físicos, psicológicos e sociais que frequentemente acompanham a idade avançada ${ }^{(3)}$.

$A$ atividade física (AF) é definida como qualquer movimento corporal, produzido pelo músculo esquelético que resulte em um pequeno aumento calórico acima do repouso e que está englobada e dividida em quatro domínios: atividades realizadas no trabalho, atividades domésticas, atividade no meio de transporte e no lazer ${ }^{(4-5)}$.

A relação entre atividade e saúde em idosos é consenso na literatura, existindo diretrizes quanto aos dias (semana normal/habitual), tempo (horas e minutos por dia e semana) e intensidade da atividade física que são benéficos para os idosos ${ }^{5}$. As AF que resultam em benefícios para a saúde são aquelas em que os idosos praticam, pelo menos, 150 minutos por semana de atividade física, no mínimo moderada, classificando-os como ativos ${ }^{(5)}$.

Este tipo de atividade, sendo realizada de forma regular, é um componente integral do envelhecimento saudável, por isso a Organização Mundial de Saúde (OMS) sugere que faça parte da rotina diária para a população idosa ${ }^{(6)}$.

A AF regular melhora o bem-estar geral e a saúde física e psicológica, ajuda a preservar as atividades de vida diárias (AVDs) e as atividades instrumentais da vida diária (AIVDs) gerando mais independência em suas vidas. $A$ atividade física específica do domínio de lazer (exercício físico e/ou esportes) auxilia no controle de condições específicas (estresse, obesidade), reduz o risco de doenças crônicas (doença coronária cardíaca, hipertensão, diabetes), minimiza as consequências de certas incapacidades e contribui na administração de condições dolorosas ${ }^{(7-8)}$.

Acredita-se que o envelhecimento bem sucedido é resultado em grande parte do estilo de vida do idoso( ${ }^{(9)}$. A prática de atividade física como promotora de independência funcional e desenvolvimento saudável é comum à maioria dos idosos, bem como o não uso de tabagismo e do etilismo, que favorece a ausência de comorbidades relacionadas e óbito precoce possibilitando que o idoso possa desfrutar de sua saúde e consequentemente obter uma melhor qualidade de vida ${ }^{(10)}$.

Para a OMS, um dos componentes mais importantes para se ter uma boa saúde é o estilo de vida adotado pelas pessoas, o qual pode ser entendido como as ações realizadas pelo indivíduo no seu dia a dia: alimentação, uso de drogas (lícitas e/ou ilícitas), prática de atividades físicas e exercícios físicos regulares, dentre outros, que são passíveis de serem modificadas. Essa mesma organização reconhece a prática de atividades físicas como um relevante meio de promoção da saúde e redução dos fatores de risco(II).

Diante o exposto, o estudo apresenta como objetivo avaliar o nível de atividade física em mulheres idosas e seus fatores associados.

\section{MÉTODO}

Trata-se de um estudo observacional-descritivo, quantitativo de corte transversal.A pesquisa foi realizada na Universidade Aberta à Terceira Idade - UNATI, localizada no Campus da Universidade Federal de Pernambuco (UFPE). Foram selecionadas 120 idosas, mas 10 se recusaram a participar da pesquisa, perfazendo um total de 110 entrevistadas. Todas as participantes frequentavam os cursos de línguas (inglês espanhol e italiano). Por realizarem esses tipos de cursos, as idosas tendem a ter um bom nível de cognição, já que o aprendizado de uma língua estrangeira infere em um melhor estado de cognição(12) como também de audição, por ser um facilitador do processo no andamento ao curso(12), por consequência não necessitaria avaliá-los.

Os dados sociodemográficos, clínicos e de estilo de vida foram consultados por meio do formulário de Matrícula da Universidade para a Terceira Idade (UNATI). A avaliação da função e satisfação sexual foi avaliada pelo Quociente Sexual Feminino (QS-F). Já o nível de atividade física foi medido pelo Questionário Internacional de Atividade Física (IPAQ) e, concluiu com a avaliação da imagem corporal com a Escala de Nove Silhuetas. Foram complementados por entrevista estruturada e elaborada pela equipe de pesquisa.

O formulário de matrícula foi constituído por perguntas que abordassem os dados pessoais (idade, moradia, nível de escolaridade, estado civil e se tem filhos),-socioeconômicos (renda mensal, situação previdenciária), comorbidades (pressão alta, diabetes, cardiopatias, deficiências hormonais e se utilizava medicamentos), estilo de vida (etilismo, tabagismo e se usa drogas ilícitas).

A versão longa do IPAQ apresenta 27 questões relacionadas com as atividades físicas, realizadas numa semana normal, com intensidade vigorosa, moderada e leve, com a duração mínima de 10 minutos contínuos, distri- 
buídas em quatro dimensões de atividade física (trabalho, transporte, atividades domésticas e lazer) ${ }^{(13)}$.

O QS-F avaliou a função e satisfação sexual feminino, compõe-se de 10 questões, que avaliam os aspectos de desejo e interesse sexual (questões I,2,8); preliminares (questão 3); excitação da mulher e sintonia com o parceiro (questões 4,5); conforto na relação sexual (questões 6,7); orgasmo e satisfação sexual (questões 9,10$)^{(14)}$.

A Escala de Nove Silhuetas proposta por Sorensen e Stunkard é constituída por desenhos de 9 silhuetas frontais ordenadas por ordem gradativa do menor para $\circ$ maior tamanho corporal. Para verificar a satisfação ou a insatisfação com a imagem corporal deve-se calcular a diferença entre a silhueta real e a silhueta ideal, apontadas pelo indivíduo. Se estiver satisfeito com a sua aparência (variação igual à zero), insatisfeito com a sua aparência (variação diferente de zero). Quando a diferença for positiva, considera-se insatisfatória por excesso de peso e quando for negativa, insatisfação por magreza. As idosas selecionavam a silhueta que parecia com ela atualmente e a que ela gostaria de ser ${ }^{(15)}$.

No primeiro contato com as idosas foi realizada a apresentação do projeto, seguida pelo preenchimento dos questionamentos supracitados. Após a apresentação, foi entregue os questionários, explicando minuciosamente cada quesito deixando aberto para caso alguém desejasse realizar algum tipo de pergunta.

Os dados foram digitados com dupla entrada no software EPI-INFO versão 3.3.2. Os resultados das variáveis categóricas foram apresentados em forma de tabelas e/ ou gráficos com suas respectivas frequências absolutas e relativas, enquanto as variáveis numéricas em medidas de tendência central e medidas de dispersão.

Para verificar possíveis associações entre as variáveis categóricas foi utilizado o teste de Qui-Quadrado de Pearson, e quando necessário, o teste exato de Fisher. As variáveis que obtiveram valores de $p$ menor ou igual a 0,05 tiveram relação com o nível de atividade física.Todas as análises serão realizadas no software SPSS (Statistical Package for the Social Sciences) para Windows, versão 13.0 .

Os procedimentos metodológicos deste estudo foram aprovados pelo Comitê de Ética em Pesquisas do Centro de Ciências da Saúde da Universidade Federal de Pernambuco, sob o número de registro $\mathrm{CAAE}$ 0।4365।8.1.0000.5208. Este artigo é um recorte advindo da dissertação da primeira autora. Sendo o consentimento dos participantes ao estudo expresso através da assinatura do termo de consentimento livre e esclarecido, mantendo seus nomes resguardados. Este artigo advém da dissertação "Função e Satisfação sexual em mulheres idosas e seus fatores associados", onde foi subdivida em diversos temas para a formação de outros trabalhos e artigos.

\section{RESULTADOS}

Foram entrevistadas 110 idosas, das quais apresentaram uma faixa etária entre 60 a 84 anos, com média de 67,17 anos e desvio padrão de 5,24 , com maior concentração de idosas entre 60 a 69 anos.

O perfil das idosas entrevistadas no que concerne a aspectos sociais, demográficos, clínico e de estilo de vida foram: nível superior completo $46(41,8 \%)$, casadas 35 (3I,8\%), tinham filhos 94 (85,5\%), aposentadas 77 (70\%), moravam sozinhas 34 (30,9\%), com renda entre um à dois salários mínimos 39 (35,5\%) (valor do SM em $2019=\mathrm{R} \$ 998$ reais). Não ingeriam álcool 90 $(84,9 \%)$ e também não fumavam 90 (84,9\%). Apenas $3(2,7 \%)$ das idosas usavam drogas ilícitas. 56 (50,9\%) não tinham hipertensão, $54(49,1 \%)$ não eram diabéticas, 8 I $(73,6 \%)$ como também a maioria não eram cardiopatias 102 (92,7\%), 91 (82,7\%) não tinham deficiência hormonal, porém $88(80 \%)$ das idosas usam medicamentos, $68(61,8 \%)$ as idosas tinham vontade de ter o corpo mais magro. Sobre a função e satisfação sexual, a maioria das idosas obteve nível de "nulo a ruim". (Tabela I)

$\mathrm{Na}$ tabela 2 mostra as variáveis que concerne a aspectos sociais, demográficos, clínico e de estilo de vida, com o nível de atividade física.

Dentre as categorias socioeconômicas apenas a situação previdenciária teve valor próximo de 0,05 , consequentemente seja provável que a maioria das idosas aposentadas são consideradas ativas. Já as variáveis de renda mensal, origem dos recursos, atividade remunerada não obtiveram relação com a atividade física.

Dentre as três categorias de estilo de vida, o teste de Fisher revelou que duas apresentava associação estatisticamente significativa com o nível de atividade física. O etilismo, foi uma das categorias que teve influencia, concluindo assim que a maioria das idosas ativas não ingeriam bebidas alcoólicas. Sobre o tabagismo, a maioria das idosas que não fumavam eram ativas (Tabela 2). A variável que não teve associação com a atividade física foi às drogas.

Dentre as comorbidades, a DM foi a variável que teve um valor de $p$ próximo a 0,05. Já a as variáveis: HAS, Cardiopatias, Deficiência Hormonal e o uso de Medicação não obtiveram nenhuma associação com a atividade física através do teste qui-quadrado de Pearson.

As variáveis: escolaridade, estado conjugal, com quem reside, filhos, sexualidade, imagem corporal não obtiveram associação estatística com a variável dependente (VD). Já as variáveis: etilismo e tabagismo tiveram associação com o nível de atividade física em idosas. 
TABELA 1 - Perfil sociodemográfico, clínico e estilo de vida das idosas frequentadoras dos cursos de línguas na UNATI - UFPE. Recife, PE, 2020

\section{Escolaridade}

De 05 a 08 anos de estudo (antigo ginásio)

De 09 a 12 anos de estudo (antigo cientifico, magistério)

Superior Completo (graduado)

Pós-Graduado

\section{Com quem reside}

Esposo (a) ou companheiro (a)

Filho (a) ou enteado (a)

Neto (a)

Irmão (a)

Outros Parentes, amigos (a)

Mora sozinha

Outros agrupamentos

\section{Situação Previdenciária}

Não aposentada

Aposentada

Pensionista

Aposentada e Pensionista

Estado Civil

Solteiro (a)

Casado (a) ou união estável

Divorciado

Viúvo

Filhos

Sim

Não

Renda Mensal

Menos de 1 salário

De 1 a 2 salários

Entre 2 a 4 salários

Mais de 4 salários

Sem Renda

HAS

Sim

Não

DM

Sim

Não

Cardiopatias

Sim

Não

Drogas

Sim

Não

Deficiência Hormonal

Sim

Não

Medicação

Sim

Não

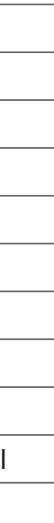


TABELA 2 - Distribuição dos dados pessoais, das comorbidades, dos hábitos de vida, da imagem corporal e da função sexual em idosas frequentadoras da UNATI. Recife, PE, 2020

\section{IPAQ Total}

Variáveis

Escolaridade

De 05 a 08 anos de estudo (antigo ginásio)

De 09 a 12 anos de estudo (antigo cientifico,

magistério)

Superior Completo (graduado)

Pós-Graduado

Filhos

Sim

Não

Estado Civil

Solteiro (a)

Casado (a) ou união estável

Divorciado Viúvo

Com quem reside

Esposo (a) ou companheiro (a)

Filho (a) ou enteado (a) Neto (a)

Irmão (a)

Outros Parentes, amigos (a)

Mora sozinha

Outros agrupamentos

Situação Previdenciária

Não Aposentada

Aposentada

Pensionista

Aposentada e pensionista

Renda Mensal

Menos de 1 salário

De 1 a 2 salários

Entre 2 a 4 salários

Mais de 4 salários

Sem Renda

Etilismo

Sim

Não

Tabagismo

Sim

Não

Drogas Ilícitas.

Sim

Não

HAS

Não

DM

Sim

Não

Cardiopatas

Sim

Não

Deficiência Hormonal

Sim

Não

Medicação

Sim

Não

Imagem Corporal

Satisfação Corporal

Ter um corpo maior (negativo)

Ter um corpo magro (positivo)

Função Sexual

Bom a Excelente

Regular a Bom

Desfavorável a Regular

Ruim a Desfavorável Nulo a Ruim
Ativa (>= $150 \mathrm{~min} / \mathrm{sem}$ ) $\mathrm{N}(\%)$

$4(66,7 \%)$

$34(85,0 \%)$

$37(80,4 \%)$

$16(88,9 \%)$

$80(85,1 \%)$

$11(68,8 \%)$

$16(72,7 \%)$

$31(88,6 \%)$

$20(83,3 \%)$

$24(82,8 \%)$

$19(90,5 \%)$

$24(88,9 \%)$

$2(66,7 \%)$

$3(75,0 \%)$

$4(80,0 \%)$

$25(73,5 \%)$

$14(87,5 \%)$

$13(86,7 \%)$

$66(85,7 \%)$

$10(9,1)$

$8(7,3)$

$5(83,3 \%)$

$34(87,2 \%)$

$24(80,0 \%)$

$26(78,8 \%)$

$2(100,0 \%)$

1 (25)

$90(84,9)$

1 (25)

$90(84,9)$

$2(66,7 \%)$

$89(83,2 \%)$

$45(83,3 \%)$

$46(82,1 \%)$

$27(93,1 \%)$

$64(79,0 \%)$

$5(62,5 \%)$

$86(84,3 \%)$

$16(84,2 \%)$

$75(82,4 \%)$

$72(81,8 \%)$

$19(86,4 \%)$

$28(30,8 \%)$

$6(6,6 \%)$

$57(62,6 \%)$

$25(27,5 \%)$

$18(19,8 \%)$

$10(11,0 \%)$

$6(6,6 \%)$

$32(35,2 \%)$

Inativo (<= $150 \mathrm{~min} / \mathrm{sem}) \quad$ p-valor ${ }^{1}$

(<= $150 \mathrm{~min} / \mathrm{sem})$
$\mathrm{N}(\%)$

$0,587^{1}$

$2(33,3 \%)$

$9(19,6 \%)$

$2(11,1 \%)$

$14(14,9 \%)$

$5(31,3 \%)$

$6(27,3 \%)$

$4(11,4 \%)$

$4(16,7 \%)$

$5(17,2 \%)$

$2(9,5 \%)$

$3(11,1 \%)$

$1(33,3 \%)$

$1(25,0 \%)$

$1(20,0 \%)$

$9(26,5 \%)$

$2(12,5 \%)$

$2(13,3 \%)$

$11(14,3 \%)$

$2(20,0 \%)$

$4(50,0 \%)$

$1(16,7 \%)$

$5(12,8 \%)$

$6(20,0 \%)$

$7(21,2 \%)$

$0(, 0 \%)$

$3(75)$

$16(15,1)$

$3(75)$

$16(15,1)$

$1(33,3 \%)$

$18(16,8 \%)$

$9(16,7 \%)$

$10(17,9 \%)$

$2(6,9 \%)$

$17(21,0 \%)$

$3(37,5 \%)$

$16(15,7 \%)$

$3(15,8 \%)$

$16(17,6 \%)$

$16(18,2 \%)$

$3(13,6 \%)$

$7(36,8 \%)$

$1(5,3 \%)$

$11(57,9)$

$2(10,5 \%)$

$4(21,1 \%)$

$1(5,3 \%)$

$2(10,5 \%)$

$10(52,6 \%)$

1 p-valor do teste exato de Fisher e 2 p-valor do teste do Qui-quadrado para comparação de proporção 
A tabela 3 descreve os aspectos da escala do IPAQ, onde se divide em: trabalho, meio de transporte, atividade física em casa, atividade física no esporte e o total. As áreas onde as idosas apresentaram maior execução de atividade física foram $\mathrm{AF}$ em casa, no esporte e no meio de transporte, representando $68,2 \%, 50 \%$ e $39,1 \%$ respectivamente. $O$ pior desempenho foi observado para as atividades físicas no trabalho com $10 \%$.

Percebe-se que poucas são as idosas que realizam atividade física no trabalho II ( $10 \%)$, sobre a atividade física no meio de transporte foram $43(39,1 \%)$, com relação à atividade física em casa (atividades vigorosas e/ou moderadas ao redor e/ou dentro da casa ou apartamento), 75 $(68,2 \%)$ das idosas eram consideradas ativas. Já a atividade física no Esporte, 55 (50\%) das idosas entrevistadas eram consideradas ativas.

Com base na tabela 4 concluímos que $82,7 \%$ das idosas que frequentam os cursos de línguas da UNATI/UFPE são consideradas ativas.

\section{DISCUSSÃO}

Pelos resultados percebe-se que as variáveis que tiveram relação com a atividade física foram: Etilismo e o Tabagismo. Contrapondo com as literaturas percebe-se que a $A F$ pode ter associação também com as variáveis: diabetes, hipertensos, cardiopatias, deficiência hormonal, medicação.

Nos resultados, a maioria das idosas eram aposentadas, corroborando com o estudo, percebeu-se que $57 \%$ dos idosos conseguiram se aposentar. Sobre aposentados e pensionistas, se somarmos dará $79 \%$ das idosas na pesquisa. Resultados semelhantes relataram que $81,7 \%$ idosas tinham aposentadoria e pensão(16).
Não foi obtido relação entre a situação previdenciária com o nível de atividade física, entretanto alguns estudos mostram que devido ao tempo disponível, advindo da aposentadoria, faz com que o idoso realize vários tipos de atividades físicas. $O$ idoso precisa preencher o tempo ocioso, consequentemente amplia em atividades o seu tempo livre ${ }^{(17)}$.

$\mathrm{Na}$ pesquisa percebeu-se que a Diabetes Mellitus não teve associação com a AF em idosas. Duarte et al (20I2) corroborando com nosso estudo, foi compreendido, que idosos diabéticos obtiveram um resultado de pouco ativo pelo questionário do IPAQ. Acrescentando que I40 relataram não praticar Exercícios Físicos (E.F.) regularmente por motivos como: por desconforto, por restrição médica e por não gostarem, por falta de tempo, por preguiça e por episódios de hipoglicemia ${ }^{(18)}$.

Nos resultados foi concluído que o etilismo e o tabagismo podem ter influência com a AF. Santos (20I4) divergindo do estudo, percebeu que não existe relação entre a AF, a ingestão de álcool e do uso de tabaco em idosos moradores de três municípios de Minas Gerais, Brasil (19). Corroborando com o estudo percebeu-se que idosos sedentários apresentaram maior probabilidade de serem fumantes ${ }^{(20-21)}$. É comprovado também que idosos ex fumantes normalmente aderem a práticas de vida mais saudáveis ${ }^{(22)}$.

Reforçando os resultados foi concluído que existe associação positiva entra a AF global e o consumo de bebidas alcoólicas ${ }^{23}$. O consumo moderado de álcool pode, entretanto, atuar como um marcador da boa condição física do idoso, indicando ausência de doença grave ou de doença que impeça o consumo de bebida alcoólica.

Sobre o nível de AF em idosas, evidenciou-se que

TABELA 3 - Descrição da escala do IPAQ em idosas frequentadoras da UNATI/ UFPE, Recife. 2020.

\begin{tabular}{|c|c|c|}
\hline IPAQ & $\mathrm{N}$ & Porcentagem (\%) \\
\hline \multicolumn{3}{|l|}{ IPAQ: Trabalho } \\
\hline Ativo (>=150 min/sem) & 11 & 10,0 \\
\hline Inativo (< $150 \mathrm{~min} / \mathrm{sem})$ & 99 & 90,0 \\
\hline \multicolumn{3}{|l|}{ IPAQ: Meio de Transporte } \\
\hline Ativo (>=150 min/sem) & 43 & 39,1 \\
\hline Inativo (<150 min/sem) & 67 & 60,9 \\
\hline \multicolumn{3}{|l|}{ IPAQ: AF em casa } \\
\hline Ativo (>=150 min/sem) & 75 & 68,2 \\
\hline Inativo (< $150 \mathrm{~min} / \mathrm{sem})$ & 35 & 31,8 \\
\hline \multicolumn{3}{|l|}{ IPAQ: AF no Esporte } \\
\hline Ativo (>=150 min/sem) & 55 & 50,0 \\
\hline Inativo (< $150 \mathrm{~min} / \mathrm{sem})$ & 50,0 & 50,0 \\
\hline Total & 110 & 100 \\
\hline
\end{tabular}

TABELA 4 - Descrição da escala do IPAQ em idosas frequentadoras da UNATI/ UFPE, Recife. 2020.

\begin{tabular}{c|c|c}
\hline IPAQ: Total & N & Porcentagem (\%) \\
\hline Ativo (>=150 min/sem) & 91 & 82,7 \\
\hline Inativo (<150 min/sem) & 19 & 17,3 \\
\hline Total & 110 & 100 \\
\hline
\end{tabular}


a maioria das idosas são consideradas ativas, principalmente nas atividades domésticas. Indo de contra ao resultado, estudo mostra que os níveis de atividade física em idosas que frequentavam o hospital de Areia, localizado também na cidade do Recife, obtiveram um nível de atividade física baixo, particularmente no domínio do lazer(24). É plausível que por a pesquisa ter sido realizada em uma Universidade Aberta para a Terceira Idade (UNATI - UFPE), subentende que as idosas sejam mais saudáveis e consequentemente tenham um bom nível de atividade física, diferente das idosas que frequentam ambientes hospitalares.

Já em uma segunda pesquisa, dos 256 idosos que eram atendidos em centros de convivência, a maioria (I86) foram considerados muito ativos, sendo um publico semeIhante ao da UNATI-UFPE e que está de acordo com os resultados desta pesquisa ${ }^{(25)}$.

Reforçando os resultados, a literatura mostra que as atividades que as idosas mais praticam são as domésticas. Vale destacar que é interessante estimular às atividades de voluntariado após a aposentadoria, para o contexto do trabalho. E para o transporte, ações de políticas públicas (melhora das calçadas, da iluminação, da segurança, dentre outros) e de conscientização dos benefícios do deslocamento ativo para a saúde. Atividades estas que são pouco realizadas nos grupos da terceira idade ${ }^{(26)}$.

De forma geral, percebe-se que a prática de atividade física na terceira idade deve ser estimulada, pois melhora a qualidade de vida relacionada ao domínio físico, à força e à flexibilidade, contribuindo para a prevenção de lesões e a independência do idoso ${ }^{(27)}$.

Uma das limitações do estudo foi entrevistar a função sexual das idosas, afinal ainda é um tabu perante esta população. Para solucionar este problema, foi realizado uma apresentação utilizando um data-show e computador, com vídeos e relatos de idosas que falavam abertamente sobre sua sexualidade. A partir daí, as entrevistadas se sentiram mais a vontade para discutir o tema proposto. Outra limitação do estudo foi o uso da escala de atividade física (IPAQ), por ser uma escala muito extensa, algumas idosas tiveram dificuldade de responder. Neste aspecto, a entrevistadora ficou aberta para auxiliar nas dúvidas que poderiam surgir e então a dificuldade foi sanada.

\section{CONCLUSÃO}

Estudos sobre a atividade física e o envelhecimento humano são de suma importância na área da Gerontologia e Geriatria, posto que permitam abrir novos espaços na prática clínica dos profissionais de saúde, sendo, necessário ser discutida junto aos idosos sobre sua saúde, considerando-se que a atividade física faz parte da promoção e prevenção em saúde, da autonomia, da independência dos idosos e consequentemente influencia em uma melhor qualidade de vida desta população.

Percebe-se que fatores socioeconômicos, morbidades, o estilo de vida e a sexualidade podem ter influência no nível de atividade física dos idosos. Porém observouse que a os fatores etilismo e tabagismo tiveram relação com a atividade física, contrapondo com a literatura que cita a influência de outras variáveis.

Dentre os tipos de atividade física incluídos no domínio do IPAQ, a que teve maior pratica entre as idosas foi à atividade física doméstica.

Durante a coleta de dados, ocorreu dificuldade entre as idosas usar a escala do IPAQ, por ser uma escala extensa e longa. Solucionamos este problema com a explicação de cada questão e ficamos abertas para dúvidas e perguntas, caso as idosas tivessem. Sobre as perguntas que abordava questões da sexualidade, incluímos uma apresentação no powerpoint sobre sexualidade e realizamos um dialogo antes de iniciar as perguntas, ocorrendo uma aproximação entre o avaliador e as idosas, auxiliando na abordagem.

Sugere-se a necessidade de novas pesquisas que avaliem a associação destes fatores com a atividade física em uma amostra maior como também em outras regiões. 


\section{REFERENCIAS}

I Ponte MKC, Cunha FMAM. Nível de atividade física na população idosa e seus benefícios: uma revisão integrativa. Sanare (Sobral). 20 I 5; I2(1):7l-7.

2 Ferreira G.L. Associações entre a atividade física, a aptidão física e a densidade mineral óssea, em indivíduos idosos. Porto: G. Ferreira. Dissertação de Mestrado na área de Atividade Física para a Terceira Idade apresentada à Faculdade de Desporto da Universidade do Porto.

2 Ansarah VW, Gobbi S. Functional fitness for aged people. The 1992 Internacional Conference on Aging and Physical Activity, Fitness \& Health. Conference Programs and Abstracts. Toronto, 2016;119.

3 Barros FF. Percepção de envelhecimento pelos idosos e atividade física. Universidade de Evora - Escola de Ciências Sociais. Dissertação de mestrado - Psicologia - Psicologia da Educação, 2020.

4 Rios DR. Minidicionário Escolar Língua Portuguesa. São Paulo: DCL, 2019.

5. Mazo GZ; Benedetti, TRB. Adaptação do questionário internacional de atividade física para idosos. Rev Bras Cineantropom Desempenho Hum 2016; I2(6):480-484.

6 Gobbi S. Atividade Física para pessoas idosas e recomendações da Organização Mundial de saúde de 1996. Revista Brasileira de Atividade Física e Saúde. 2017;2(2).

7 Ansarah VW, Gobbi S. Functional fitness for aged people. The 1992 Internacional Conference on Aging and Physical Activity, Fitness \& Health. Conference Programs and Abstracts. Toronto, 2016:119.

8 Bodoyoy JL, Blair SN. Aging and Exercise: A health perspective. Journal of Aging and Physical Activity, 20 I 5;2:243-260.

9 Silva T. Freitas R. Avaliação da capacidade física e quedas em idosos ativos e sedentários da comunidade. Rev Bras. Clin Med. 2015.

I0 Zaitune, M. Barros, M. Fatores associados ao tabagismo em idosos: inquérito de saúde no estado de São Paulo (ISA-SP). Cad. Saúde Pública, Rio de Janeiro, 2017;28(3):583-595.

I I Organização Mundial de Saúde (BR). O papel da atividade física no Envelhecimento saudável. Florianópolis, 2016.

12 Porto MAR. Tempo cognitivo e tempo social nas aulas de inglês para a envelhescência e terceira idade. Editora Edgard Blücher Ltda. 2018.

I 3 Benedetti TRB,Antunes PC, Rodriguez-Añez CR, Mazo GZ, Petroski EL. Reprodutibilidade e validade do Questionário Internacional de Atividade Física (IPAQ) em homens idosos Rev Bras Med Esporte. 20I7;(I3) I.

14 Abdo CHN. Quociente sexual feminino: um questionário brasileiro para avaliar a atividade sexual da mulher. Diagn Tratamento. 2009; I4(2):89-I.

I5 Stunkard AJ; Sorensen T; Schulsinger F. Use of the Danish Adoption Register for the study of obesity and thinness. In: Kety SS, Rowland LP, Sidman RL, Matthysse SW. The genetics of neurological and psychiatric disorders. New York: Raven Press; 2017; I I5-20.

16 Teixeira L. Saúde e qualidade de vida na terceira idade. Textos Envelhecimento 20I4; I (I).

17 Moura GA, Souza LK. Autoimagem, socialização, tempo livre e lazer: quatro desafios à velhice*. Textos \& Contextos (Porto Alegre), 2016; I I (I): I 72- I83.

I 8 Duarte CK,Almeida JC, Merker AJS, Brauer FO, Rodrigues TC. Nível de atividade física e exercício físico em pacientes com diabetes mellitus. Rev Assoc Med Bras 2015;58(2):2I5-22I.

19 Santos AS, Viana DA, Souza MC, Meneguci J, Silveira RE, Silvano CM, Rodrigues LR, Damião R. Atividade Física, Álcool e Tabaco entre Idosos. REFACS (online) 20I5;2(I):06-I3.

20 Blair SN. Physical activity, epidemiology, public health and the American College of Sports Medicine. Med Sci Sports Exerc 2013; 35: I463.

2I Bowles HR, Fitzgerald SJ, Morrow JR, Jackson AW, Blair SN. Construct validity of self-reported historical physical activity. Am J Epidemiol 2014;160:279-86.

22 Monteiro CA, Conde WL, Matsudo SM, Matsudo VR, Bonseñor IM, Lotufo PA.A descriptive epidemiology of leisuretime physical activity in Brazil, 1996-1997. Rev Panam Salud Pública 20। 3; I4: 246-54.

23. Woo J, Ho SC, Yu AL. Lifestyle factors and health outcomes in elderly Hong Kong Chinese aged 70 years and over. Gerontology 2015;48:234-40.

24 Lima RA, Silvestre CMSMF, SmethurstWS, Barros MVG. Nível de atividade física em idosos com doença de Alzheimer mediante aplicação do IPAQ e de pedômetros. Revista Brasileira de Atividade Física \& Saúde. 2016;15(3).

25 Mazo GZ, Liposcki DB,Ananda C E Prevê D. Incidência de quedas, atividade física e saúde dos idosos.Rev. bras. Fisioter.20 I 7; I | (6).

26 Benetti TRB, Mazo GZ, Borges LJ. Condições de saúde e nível de atividade física em idosos participantes e não participantes de grupos de convivência de Florianópolis. Ciência \& Saúde Coletiva. 2015;17(8):2087-2093.

27 Silva MF, Goulart NBA, Lanferdini FJ, Marcon M, Dias CP. Relação entre nível de atividade física e qualidade de vida em idosos. Rev. Bras. Geriatr. Gerontol. 20 I2; I5(4):635-642.

Recebido: 2020-09-14

Aceito: 2020-II-09 